\title{
Planetesimal and Protoplanet Dynamics in a Turbulent Protoplanetary Disk: Ideal Unstratified Disks
}

\author{
Chao-Chin Yang \\ Department of Astronomy, University of Illinois, Urbana, IL 61801 \\ Department of Astrophysics, American Museum of Natural History, New York, NY 10024 \\ cyang@amnh.org \\ Mordecai-Mark Mac Low \\ Department of Astrophysics, American Museum of Natural History, New York, NY 10024 \\ mordecai@amnh.org \\ and \\ Kristen Menou \\ Department of Astronomy, Columbia University, New York, NY 10027 \\ kristen@astro.columbia.edu
}

\begin{abstract}
The dynamics of planetesimals and planetary cores may be strongly influenced by density perturbations due to magnetorotational turbulence in their natal protoplanetary gas disks. Using the local shearing box approximation, we perform numerical simulations of planetesimals moving as massless particles in a turbulent, magnetized, unstratified gas disk. Our fiducial disk model has turbulent accretion characterized by a Shakura-Sunyaev viscosity parameter of $\alpha \sim 10^{-2}$, with root-mean-square density perturbations of $\sim 10 \%$. We present detailed characterizations of the statistical evolution of particle orbital properties in our simulations. Contrary to recent claims, we find that planetesimals should be safe from turbulence-induced collisional destruction, except perhaps for kilometer-sized objects situated in the outer regions ( $~ \gtrsim 30 \mathrm{AU}$ ) of young protoplanetary disks. We confirm earlier results based on local models indicating that type-I migration probably dominates over diffusive migration by stochastic torques for most objects in the planetary core and terrestrial planet mass range. Diffusive migration remains important for objects in the mass range of kilometer-sized planetesimals. Discrepancies in the derived magnitude of turbulence between local and global simulations of magnetorotationally unstable disks remains a major open issue, with important consequences for planet formation scenarios.
\end{abstract}


Subject headings: Accretion, accretion disks — methods: numerical — MHD — planetary systems: formation — planetary systems: protoplanetary disks — turbulence

\section{INTRODUCTION}

The core accretion scenario is a key ingredient of our current theory for planet formation (see reviews by Lissauer 1993; Papaloizou \& Terquem 2006, and references therein). In this model, solid bodies ranging from micron-sized dust grains to kilometer-sized planetesimals are the building blocks from which planetary cores assemble and ultimately form giant planets orbiting stars. The dynamics of planetesimals and planetary cores may be strongly influenced by their natal protoplanetary gas disks. In particular, type-I migration due to angular momentum exchange with the gas disk could drive a planetary core into the host star in a short timescale (e.g., Goldreich \& Tremaine 1980; Ward 1997; Tanaka et al. 2002; Menou \& Goodman 2004) compared to a typical disk lifetime of $\lesssim 10$ Myr (e.g., Hartmann et al. 1998; Sicilia-Aguilar et al. 2006; Hillenbrand 2008). This remains one of the major obstacles to planet formation in the core accretion scenario (see, however, Paardekooper \& Mellema 2006; Kley \& Crida 2008; Paardekooper \& Papaloizou 2009, for a possible solution).

Turbulence must exist in young protoplanetary disks for accretion to occur at observed rates. The most likely cause of this turbulence is that disks are unstable to linear perturbations if they are weakly magnetized (Balbus \& Hawley 1991). Although the magnetorotational instability (MRI) is the most promising mechanism to drive turbulence in protoplanetary disks, the numerical convergence of simulations with increasing resolution continues to be an open issue. It has been shown that MRI-driven turbulence in ideal, magnetized, unstratified disks without mean magnetic fields decreases with increasing resolution and might be negligible when resolution is high and numerical dissipation is small (Fromang \& Papaloizou 2007; Pessah et al. 2007). To maintain a nonzero, convergent level of turbulent accretion, simulated disks might require explicit dissipation (e.g., Fromang et al. 2007; Lesur \& Longaretti 2007) or nonzero magnetic flux (e.g., Guan et al. 2009; Simon et al. 2009). Furthermore, the conductivity near the mid-plane of a protoplanetary disk may be too low for the MRI to operate, at least over some range of radii, resulting in a quiescent region known as a dead zone (e.g., Gammie 1996; Sano et al. 2000; Fromang et al. 2002; Semenov et al. 2004; Ilgner \& Nelson 2006; Turner et al. 2007). Disks containing dead zones would, nevertheless, be stirred by turbulent regions near the disk surface.

The turbulent nature of protoplanetary disks might lead to scenarios for the formation and migration of planetesimals and planetary cores that are rather different from those suggested for the better-studied case of a laminar disk. The turbulence driven by the MRI causes density enhancements significant enough to exert gravitational torques that turn the orbital motion of low-mass objects into a random walk (Laughlin et al. 2004, hereafter LSA04; Nelson \& Papaloizou 2004). It has been shown that radial excursions and eccentricities of planetesimals or protoplanets could be excited through such a process (Nelson 2005, hereafter N05; Ogihara et al. 2007, hereafter OIM07). 
Since some protoplanets could diffuse their way radially outward in the process, type-I migration might be effectively delayed and some objects might more easily survive past the gas disk depletion (Johnson et al. 2006, hereafter JGM06; Adams \& Bloch 2009). Even if the disk contains a dead zone, Oishi et al. (2007, hereafter OMM07) have shown that low-mass objects within the dead zone still experience some of the turbulent torques generated by the active layers. Ida et al. (2008, hereafter IGM08) suggest, on the other hand, that the velocity dispersion of planetesimals excited by hydromagnetic turbulence might be so strong that kilometer-sized objects suffer from collisional destruction.

The survivability of planetesimals or protoplanets under type-I migration or collisional destruction sensitively depends on their orbital dynamics in a turbulent gas disk. Previous direct orbital integrations of planetesimals or protoplanets embedded in a turbulent gas disk were generally conducted in global disk models (LSA04; N05; OIM07; IGM08). In contrast to global disk models, it can be advantageous to employ the local shearing box approximation (e.g., Goldreich \& Lynden-Bell

1965; Hawley et al. 1995; (Brandenburg et al. 1995) because of its high resolving power on turbulence structures and the possibility to integrate for long times. Nelson \& Papaloizou (2004) and OMM07 first measured the stochastic torques generated by hydromagnetic turbulence at the center of a local shearing box. In this paper, we further pursue direct orbital integration of planetesimals moving as massless particles under the gravitational influence of MRI-driven turbulence in a local shearing box. We focus our attention on unstratified disks in the context of ideal magnetohydrodynamics (MHD). To maintain a nonzero, numerically convergent level of stochastic perturbations driven by the MRI, we impose a constant vertical magnetic flux. We describe our numerical models in $\$ 2$ and present the simulations in $₫ 3$, along with statistical analyses of the disk properties and the planetesimal orbits. In 4 , we use our results to revisit the issue of survivability of planetesimals and planetary cores, before reaching our conclusions in $\$$,

\section{NUMERICAL MODELING}

We use the parallelized, cache-efficient, Pencil Code described by Brandenburg \& Dobler (2002). It solves the non-ideal MHD equations by sixth-order finite differences in space and third-order Runge-Kutta steps in time. The induction equation is solved using the magnetic vector potential $\boldsymbol{A}$ so that zero divergence of magnetic field $\boldsymbol{B}$ is guaranteed at all time. To save memory usage, the Runge-Kutta time integration is performed using the $2 N$-method (Williamson 1980). The scheme is not written in conservative form. Instead, conserved quantities like total mass are monitored to evaluate the quality of the solution. In the following subsections, we describe the equations assumed in our models as well as the numerical constructs. 


\subsection{Magnetohydrodynamics}

We use the local shearing box approximation (e.g., Goldreich \& Lynden-Bell 1965; Brandenburg et al. 1995; Hawley et al. 1995) to simulate a small Cartesian box carved out of a Keplerian disk at a large distance from the host star. The center of the box corotates with the disk at Keplerian angular speed $\Omega_{K}$, the $x$-axis is directed radially, and the $y$-axis is directed azimuthally. The vertical component of gravity from the host star is ignored and thus the disk is unstratified. We impose a vertical, external magnetic field $\boldsymbol{B}_{\text {ext }}=B_{\text {ext }} \hat{\boldsymbol{z}}$ to maintain a finite magnetic flux. The MHD equations then become

$$
\begin{aligned}
\partial_{t} \rho-\frac{3}{2} \Omega_{K} x \partial_{y} \rho+\nabla \cdot(\rho \boldsymbol{u})= & f_{D}+\nabla \cdot\left(\nu_{s} \nabla \rho\right), \\
\partial_{t} \boldsymbol{u}-\frac{3}{2} \Omega_{K} x \partial_{y} \boldsymbol{u}+\boldsymbol{u} \cdot \nabla \boldsymbol{u}= & -\frac{1}{\rho} \nabla p+\left(2 \Omega_{K} u_{y} \hat{\boldsymbol{x}}-\frac{1}{2} \Omega_{K} u_{x} \hat{\boldsymbol{y}}\right)+\frac{1}{\rho} \boldsymbol{J} \times\left(\boldsymbol{B}+\boldsymbol{B}_{\mathrm{ext}}\right) \\
& +\boldsymbol{f}_{V}+\frac{1}{\rho} \nabla\left(\nu_{s} \rho \nabla \cdot \boldsymbol{u}\right), \\
\partial_{t} \boldsymbol{A}-\frac{3}{2} \Omega_{K} x \partial_{y} \boldsymbol{A}= & \frac{3}{2} \Omega_{K} A_{y} \hat{\boldsymbol{x}}+\boldsymbol{u} \times\left(\boldsymbol{B}+\boldsymbol{B}_{\mathrm{ext}}\right)+\boldsymbol{f}_{R}-\eta_{s} \boldsymbol{J},
\end{aligned}
$$

in which $\rho$ is gas density, $\boldsymbol{u}$ is gas velocity relative to the background shear flow, $p$ is gas pressure, $\boldsymbol{J}=\nabla \times \boldsymbol{B} / \mu_{0}$ is the electric current density, $\boldsymbol{B}=\nabla \times \boldsymbol{A}$, and $\mu_{0}$ is permeability. The terms $f_{D}$, $\boldsymbol{f}_{V}$, and $\boldsymbol{f}_{R}$, and those containing scalar variables $\nu_{s}$ and $\eta_{s}$ are numerical dissipation terms needed to stabilize the scheme, which are described below. They are needed to resolve shocks, and because the difference scheme formally has vanishing dissipation. We assume an isothermal equation of state, $p=c_{s}^{2} \rho$, where $c_{s}$ is the isothermal speed of sound.

The mass diffusion term $\nabla \cdot\left(\nu_{s} \nabla \rho\right)$ in the continuity equation (11) and the bulk viscosity term $\nabla\left(\nu_{s} \rho \nabla \cdot \boldsymbol{u}\right) / \rho$ in the momentum equation (2) are implemented to broaden shocks. The artificial kinematic viscosity $\nu_{s}$ is of von Neumann type (c.f., Haugen et al. 2004; Lvra et al. 2008):

$$
\nu_{s}= \begin{cases}-h^{2} \nabla \cdot \boldsymbol{u}, & \text { if } \nabla \cdot \boldsymbol{u}<-c_{s} / 4 h, \\ 0, & \text { otherwise, }\end{cases}
$$

where $h$ is grid spacing. It is smoothed by taking a maximum over nearest neighbors and then convolved with a Gaussian kernel having a standard deviation of $h$. Note that the threshold for the velocity divergence is set for eliminating artificial diffusion where hydrodynamic shocks are unlikely to be present.

We also include the Ohmic term $-\eta_{s} \boldsymbol{J}$ in the induction equation (3) to broaden strong current sheets. The numerical resistivity $\eta_{s}$ assumes the same form as used by Nitta et al. (2001) but with a lower cutoff:

$$
\eta_{s}= \begin{cases}\mu_{0} h v_{A}, & \text { if } v_{A}>8 c_{s}, \\ 0, & \text { otherwise }\end{cases}
$$

where $v_{A}=\left|\boldsymbol{B}+\boldsymbol{B}_{\text {ext }}\right| / \sqrt{\mu_{0} \rho}$ is the Alvén speed. Although simple, this form may perform better to resolve sharp magnetic structures than a resistivity proportional to the magnitude of current 
density itself (Fragile et al. 2005). We apply a lower cutoff in equation (5) to only treat regions where fast magnetic reconnection may occur.

In addition to applying artificial diffusions addressing shocks and current sheets, we also implement hyperdiffusion $f_{D}$, hyperviscosity $\boldsymbol{f}_{V}$, and hyperresistivity $\boldsymbol{f}_{R}$ in the respective MHD equations (11)-(3) (e.g., Haugen \& Brandenburg 2004; Johansen \& Klahr 2005):

$$
f_{D}=\nu_{3} \nabla^{6} \rho, \quad \boldsymbol{f}_{V}=\nu_{3}\left(\nabla^{6} \boldsymbol{u}+\mathbf{S} \cdot \nabla \ln \rho\right), \quad \text { and } \quad \boldsymbol{f}_{R}=\nu_{3} \nabla^{6} \boldsymbol{A},
$$

where $\nu_{3}$ is a constant and the tensor $\mathbf{S}$ is defined by $S_{i j} \equiv \partial_{j}^{5} u_{i}$. These terms are intended to selectively dissipate features only at high wavenumbers and thus increase the inertial range of the modeled turbulence. We adjust the coefficient $\nu_{3}$ such that the mesh Reynolds number

$$
\operatorname{Re} \equiv \frac{u_{\max }}{\nu_{3}}\left(\frac{h}{\pi}\right)^{5} \lesssim 1
$$

where $u_{\max }$ is the maximum speed relative to the background shear flow. This criterion states that dissipation at the Nyquist frequency should be comparable to or stronger than gas advection.

\subsection{Particle Dynamics}

In this work, we consider particles of zero mass to study the effects of hydromagnetic turbulence on the orbital properties of planetesimals. Although simple, this offers a good approximation for kilometer-sized planetesimals as they are large enough that gaseous drag force can be neglected, but small enough that type-I migration does not dominate. For the approximation to be valid, the mass of a particle must lie between roughly $10^{14} \mathrm{~g}$ and $10^{26} \mathrm{~g}$, corresponding to a size range of about 0.1-1000 km (OMM07). Furthermore, if the action of hydromagnetic turbulence on the particles is separable from effects due to other interactions, our measurements can be applied to all stages of planet formation.

In our models, therefore, we consider particles moving under only the gravitational influence of the host star and that of the protoplanetary gas disk. We ignore drag forces between particles and gas and the gravity of the particles. Because neither the gas disk nor other particles experience gravitational forces from the particles, no migration torques act. Given deterministic Keplerian shear flow and epicycle motions, deviations in particle trajectories due to turbulent fluctuations in the gas can easily be isolated.

Under these assumptions, the equations of motion for each particle become

$$
\begin{aligned}
\frac{\mathrm{d} \boldsymbol{x}_{p}}{\mathrm{~d} t} & =\boldsymbol{u}_{p}-\frac{3}{2} \Omega_{K} x_{p} \hat{\boldsymbol{y}} \\
\frac{\mathrm{d} \boldsymbol{u}_{p}}{\mathrm{~d} t} & =\left(2 \Omega_{K} u_{p, y} \hat{\boldsymbol{x}}-\frac{1}{2} \Omega_{K} u_{p, x} \hat{\boldsymbol{y}}\right)-\nabla \Phi .
\end{aligned}
$$


The vector $\boldsymbol{x}_{p}$ is the position of the particle in the shearing box, while $\boldsymbol{u}_{p}$ is the velocity of the particle relative to the background shear flow. The scalar variable $\Phi$ is the gravitational potential of the gas, which is the solution of the Poisson equation (Goldreich \& Lynden-Bell 1965):

$$
\left[\left(\partial_{x}-\frac{3}{2} \Omega_{K} x t \partial_{y}\right)^{2}+\partial_{y}^{2}+\partial_{z}^{2}\right] \Phi=4 \pi G \rho
$$

where $G$ is the gravitational constant.

The boundary conditions adopted for equation (9) are sheared periodic, i.e., the system is periodic after shift in the $y$-direction with the background shear flow (Hawley et al. 1995). Rather than interpolating $\rho(x, y, z)$ in real space to obtain sheared periodicity, we solve equation (9) in Fourier space and apply corresponding phase-shifts to each mode (see Johansen et al. 2007, Supplementary Information).

The position $\boldsymbol{x}_{p}$ and velocity $\boldsymbol{u}_{p}$ of each particle is updated by solving the equations of motion (88) simultaneously with the third-order Runge-Kutta steps for the MHD equations. In addition to the Courant conditions set by the MHD equations, the timestep is limited by the absolute maximum of equation (8a) such that no particles can cross more than half the zone size in one timestep. We compute the gradient of the potential $\nabla \Phi$ on the grid after solving equation (9) and then quadratically interpolate it to the position of each particle in the calculation of equation (8b).

\subsection{Code Units and Scaling Relations}

We define the length and the time units as the vertical scale height $H$ and the orbital period $P=2 \pi / \Omega_{K}$, respectively. Since vertical hydrostatic equilibrium of isothermal gas requires that $H=\sqrt{2} c_{s} / \Omega_{K}$, the speed of sound is fixed at $c_{s}=\pi \sqrt{2}$. Note that this choice makes the system invariant with temperature.

We adopt two mass units such that $\rho_{0}=\left(4 \pi G P^{2}\right)^{-1}$ and $\rho_{0}=\left(G P^{2}\right)^{-1}$ for a low-mass and a high-mass disk, respectively, where $\rho_{0}$ is the uniform initial gas density. For these two disk models, the Toomre $Q$ parameter for the gas is $Q_{g}=c_{s} \Omega_{K} / \pi G \Sigma=63$ and 5.0, respectively, where $\Sigma=\sqrt{\pi} \rho_{0} H$ is the column density 1 The gas disks in our models are gravitationally stable and thus we ignore gas self-gravity. For convenience, we define a dimensionless parameter $\xi \equiv 4 \pi G \rho_{0} P^{2}=$ $4(2 \pi)^{3 / 2} / Q_{g}$ as a measure of the strength of disk gravity. For our low-mass and high-mass disks, $\xi=1$ and $4 \pi$, respectively.

In physical units, $\rho_{0}$ is given by the following scaling relation:

$$
\rho_{0}=\left(1.2 \times 10^{-9} \mathrm{~g} \mathrm{~cm}^{-3}\right) \xi\left(\frac{P}{\mathrm{yr}}\right)^{-2}=\left(1.2 \times 10^{-9} \mathrm{~g} \mathrm{~cm}^{-3}\right) \xi\left(\frac{M_{\star}}{M_{\odot}}\right)\left(\frac{R}{\mathrm{AU}}\right)^{-3},
$$

\footnotetext{
${ }^{1}$ Strictly speaking, this relation only holds for stratified disks. Comparison between stratified and unstratified disk models will be made in a subsequent study.
} 
where $M_{\star}$ is the mass of the host star and $R$ is the distance of the shearing box to the star. The corresponding column density is

$$
\Sigma=\left(1.5 \times 10^{3} \mathrm{~g} \mathrm{~cm}^{-2}\right) \xi\left(\frac{c_{s}}{10^{5} \mathrm{~cm} \mathrm{~s}^{-1}}\right)\left(\frac{P}{\mathrm{yr}}\right)^{-1} .
$$

These scaling relations describe families of disk models to which our results apply. In particular, at 1 AU around a solar-type star, the column density of our low-mass disk is roughly consistent with that of the classical minimum mass solar nebula (MMSN; Havashi 1981).

Finally, the units of magnetic field and vector potential are $\mu_{0}^{1 / 2} \rho_{0}^{1 / 2} H P^{-1}$ and $\mu_{0}^{1 / 2} \rho_{0}^{1 / 2} H^{2} P^{-1}$, respectively. We arbitrarily set the permeability $\mu_{0}=1$. The magnetic energy density associated with $B=1$ is then $1 /\left(4 \pi^{2}\right)$ of the initial pressure $p_{0}=c_{s}^{2} \rho_{0}$.

\subsection{Initial and Boundary Conditions}

The initial conditions for our models are the following. The gas density is uniform $\left(\rho=\rho_{0}\right)$ while the magnetic vector potential is set to zero $(\boldsymbol{A}=0)$. An external vertical magnetic field is imposed, ranging from $B_{\text {ext }}=0.01$ to 0.64 . The corresponding plasma $\beta \equiv 2 \mu_{0} \rho c_{s}^{2} / B^{2}$ ranges from $3.9 \times 10^{5}$ to 96 . Gaussian noise in gas velocity of amplitude $10^{-3}$ is imposed to seed the MRI.

As noted in Section 2.2, the boundary conditions for all dynamical variables are sheared periodic and we find values for the ghost zones with a Fourier method. We fix the computational domain at $(2,2,2) H$. The highest resolution we use is 64 grid points per disk scale height.

We uniformly distribute 32,768 particles in the entire computational domain. We do not allow them to move until a time $t=t_{0}$ after the hydromagnetic turbulence has saturated and approached a statistically steady state. For the case of $B_{\text {ext }}=0.08\left(\beta_{\text {ext }}=6.2 \times 10^{3}\right)$, we choose $t_{0}=20 P$ (see (3.2). Then the particles are set to initially move relative to the background shear flow such that they have an initial eccentricity of $e_{0}$ and start at the apogee of their orbits, i.e.,

$$
\boldsymbol{u}_{p, 0}=-\frac{1}{2} H \Omega_{K}\left(\frac{e_{0}}{H / R}\right) \hat{\boldsymbol{y}}
$$

(see Appendix $(\mathrm{A})$. We wrap a particle around when it moves beyond any of the six boundary planes.

We remark that each model presented in the following section is just one realization of the stochastic nature of the turbulence. The similarity in particle orbital evolutions found across several models are due to closeness of the random number sequences and thus similar initial conditions for the gas. 


\section{SIMULATION RESULTS}

\subsection{Convergence of Turbulence Properties}

We first present a study of the convergence with increasing numerical resolution of the properties of the turbulence important to our work. Figure 1 plots density perturbation $\Delta \rho / \rho_{0}$, inverse plasma $\beta$, and the Shakura \& Sunvaev (1973) $\alpha$-parameter as a function of time $t$ for disks with an external magnetic field of $B_{\text {ext }}=0.08\left(\beta_{\text {ext }}=6.2 \times 10^{3}\right)$ at resolutions up to 64 grid points per disk scale height $H$, where $\Delta \rho \equiv \rho-\rho_{0}$. The density perturbation $\Delta \rho / \rho_{0}$ shown is the root-mean-square value over the computational domain while the inverse plasma $\beta$ shown is the volume average value. The $\alpha$-parameter is calculated from the combined effects due to the Reynolds and Maxwell shear stresses (e.g., Brandenburg 1998):

$$
\alpha=\frac{\sqrt{2}}{3} \frac{\left\langle\rho u_{x} u_{y}-B_{x} B_{y} / \mu_{0}\right\rangle}{\rho_{0} c_{s}^{2}},
$$

where the bracket \langle\rangle denotes the volume average over the entire computational domain. As shown in Figure 1, the MRI saturates and remains roughly steady after about $t_{0}=20 P$. After saturation, all three properties exhibit small changes between different resolutions, with a slight trend of increasing $\alpha$ with increasing resolution. The properties of the saturated turbulence appear to converge to a nonzero level, as opposed to disks without net magnetic flux (e.g., Fromang \& Papaloizou 2007). We expect yet smaller changes in these properties for calculations with higher resolutions than have been achieved here.

\subsection{Vertical Net Flux Dependence}

Figure 2 plots the same properties of the saturated turbulence as a function of the external vertical magnetic field, represented by the inverse plasma $\beta$. They are volume averaged as described above, and then time-averaged over a period of at least $20 P$ after saturation. Also included in the figure are the time variation of these properties, as indicated by the error bars. We confirm the general trend of increasing turbulence activity with increasing uniform vertical field (e.g., Hawley et al. 1995; Sano et al. 2004; Johansen et al. 2006). Numerical convergence can be seen over the range of the field strength we have explored. In addition to the turbulent transport often discussed in the literature, we also report the dependency between density perturbation and external field in Figure 2, which may be more relevant to the orbital dynamics of particles moving in these disks.

We emphasize that by varying the net vertical magnetic flux through a disk, a wide range of turbulent viscosity values can be obtained, as suggested by numerous previous works as well as Figure 2, To best represent typical protoplanetary accretion disks, we adopt a fiducial disk model with $B_{\text {ext }}=0.08\left(\beta_{\text {ext }}=6.2 \times 10^{3}\right)$. As shown in Figures 1 and 2, this model gives a turbulent accretion of $\alpha \sim 10^{-2}$, which is consistent with current estimates for disks around typical T Tauri stars (e.g., Hartmann et al. 1998, 2006). Note that in our fiducial model, the root-mean-square 


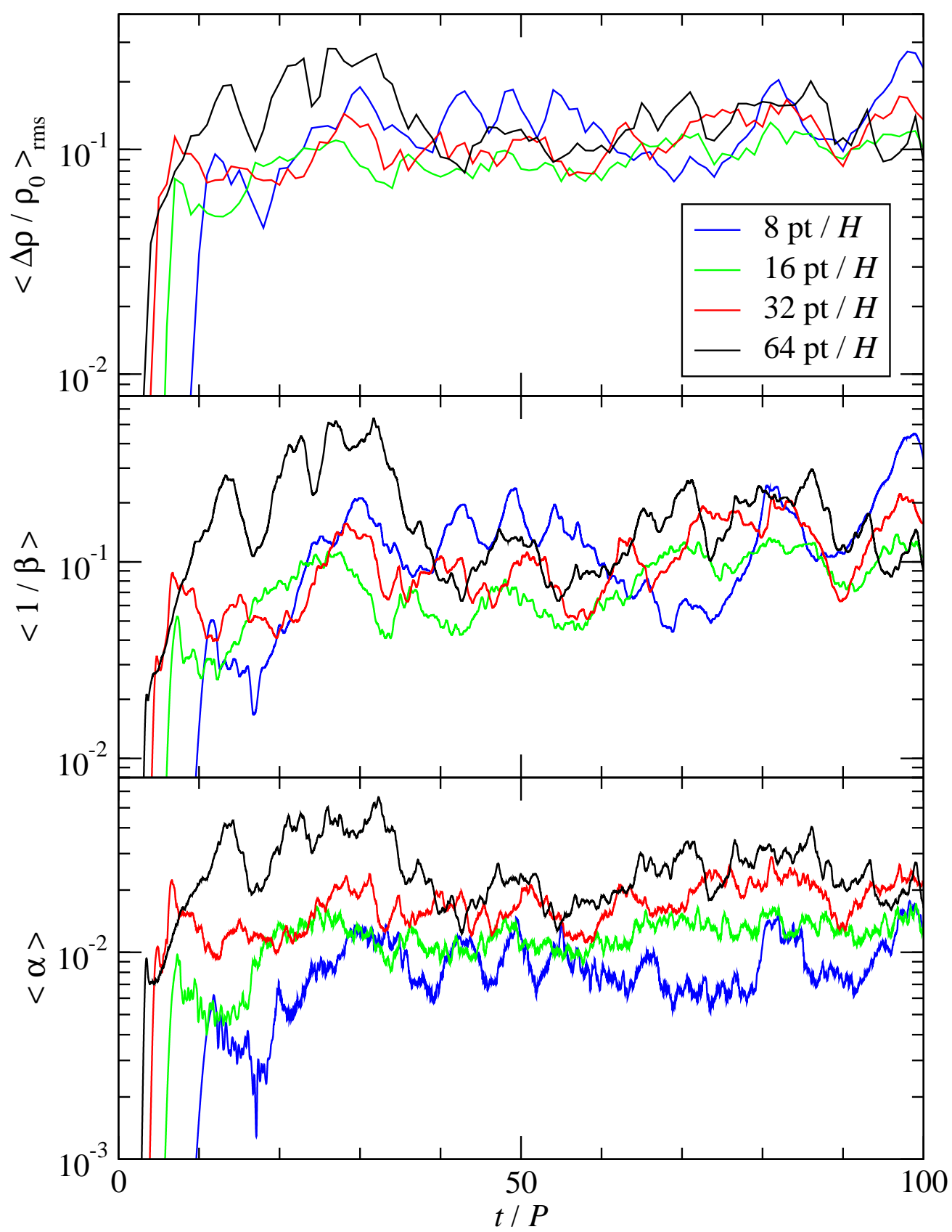

Fig. 1. - Density perturbation $\Delta \rho / \rho_{0}$, inverse plasma $\beta$, and $\alpha$-parameter as a function of time $t$ (in units of orbital period $P$ ) for an external vertical magnetic field of $B_{\text {ext }}=0.08\left(\beta_{\text {ext }}=6.2 \times 10^{3}\right)$. All properties are volume-averaged over the whole computational domain with the root-mean-square value of $\Delta \rho / \rho_{0}$ being given. Results are shown for resolutions up to 64 points per scale height $H$. 


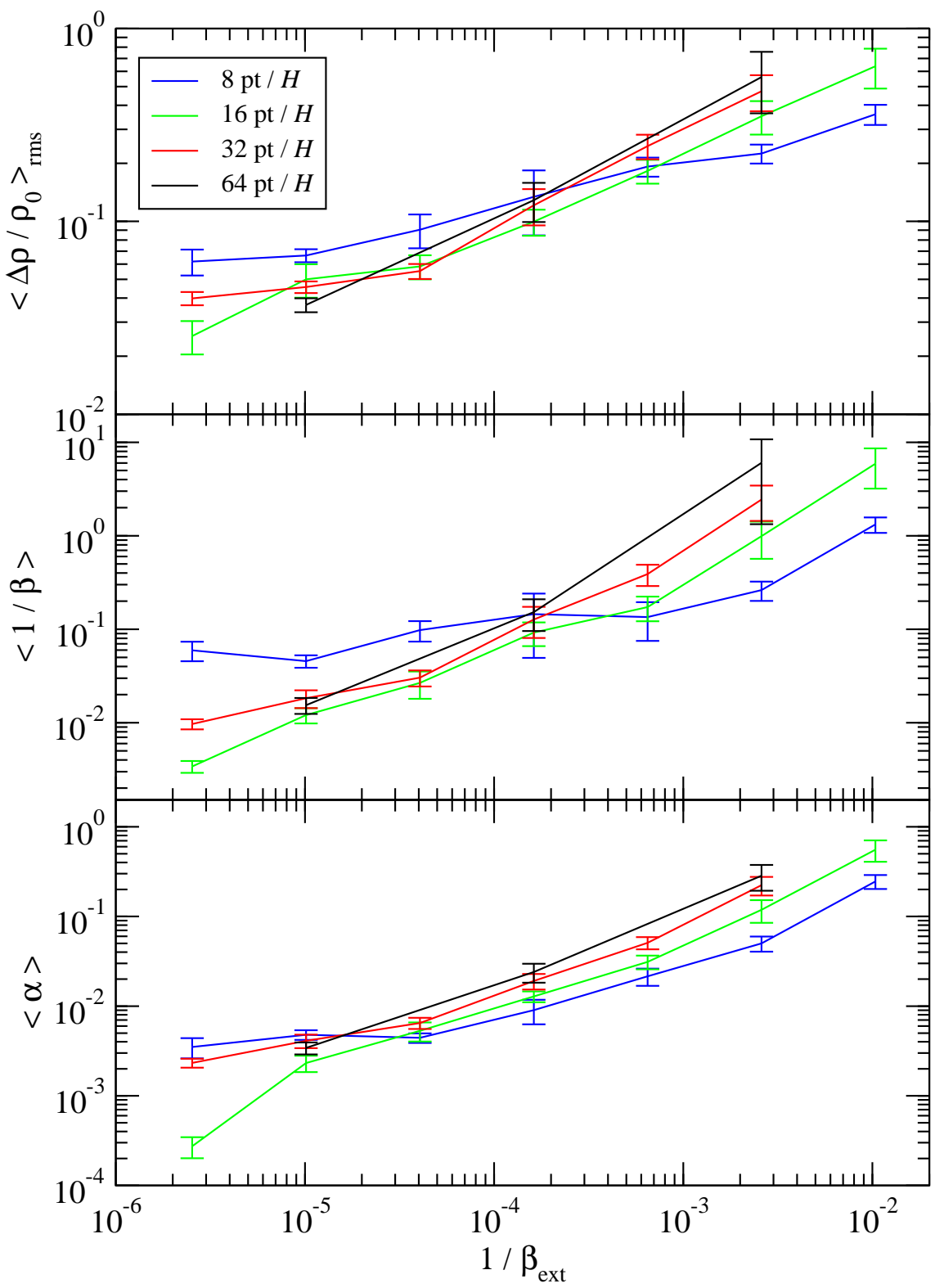

Fig. 2.- Density perturbation $\Delta \rho / \rho_{0}$, inverse plasma $\beta$, and $\alpha$-parameter as a function of external magnetic field in terms of inverse plasma $\beta$. All properties are volume-averaged over the whole computational domain as well as time-averaged over an interval of at least 20 orbital periods after saturation of the MRI. Results are shown for resolutions up to 64 points per scale height $H$. The error bars denote $1 \sigma$ in time variation around the volume-averaged properties.

density perturbation of the gas is on the order of $\sim 10 \%$. In the following subsections, we report orbital dynamics of zero-mass particles moving in our fiducial model at our maximum resolution of 


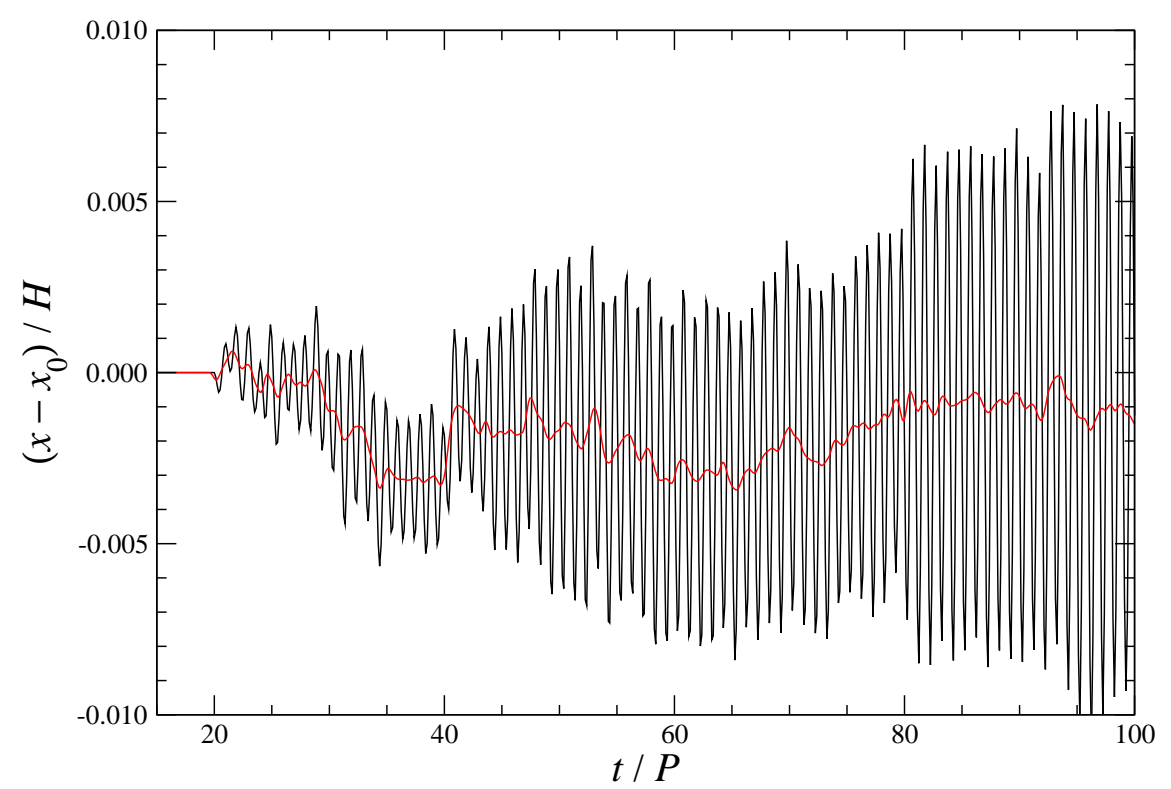

Fig. 3.- Radial motion of one representative particle with initial eccentricity $e_{0}=0$ moving in a low-mass disk. The black line shows its radial displacement $x-x_{0}$ from initial position $x_{0}$ as a function of time $t$. The red line shows the corresponding radial drift, defined as the running average over one epicycle, i.e., one orbital period $P$.

64 grid points per disk scale height.

\subsection{Motion of a Single Particle}

As demonstrated in Figure 3, a particle moving under gravity of the turbulent gas undergoes epicycle motion horizontally as well as continuous change in its mean radius. We define the radial drift of each particle as $\Delta x \equiv \bar{x}-x_{0}$, where $\bar{x}$ is the mean radial position over one orbital period (as examplified by the red line in Fig. 3) and $x_{0}$ is the initial radial position. Given that $x \ll R$, the eccentricity of each particle can be approximated by $e \approx\left(x_{\max }-x_{\min }\right) / 2 R$, where $x_{\max }$ and $x_{\min }$ are the maximum and the minimum radial positions in one epicycle, respectively. With these two quantities, we can measure the orbital migration and eccentricity change of planetesimals induced by hydromagnetic turbulence.

Figure 4 shows the change of radial drift and eccentricity with time for four randomly selected particles. It is evidently a stochastic process and the final outcome can be quite different with slightly different initial conditions. Nevertheless, statistical methods can be employed to quantify the orbital properties. We discuss the statistical evolution of these properties in the following subsections. 


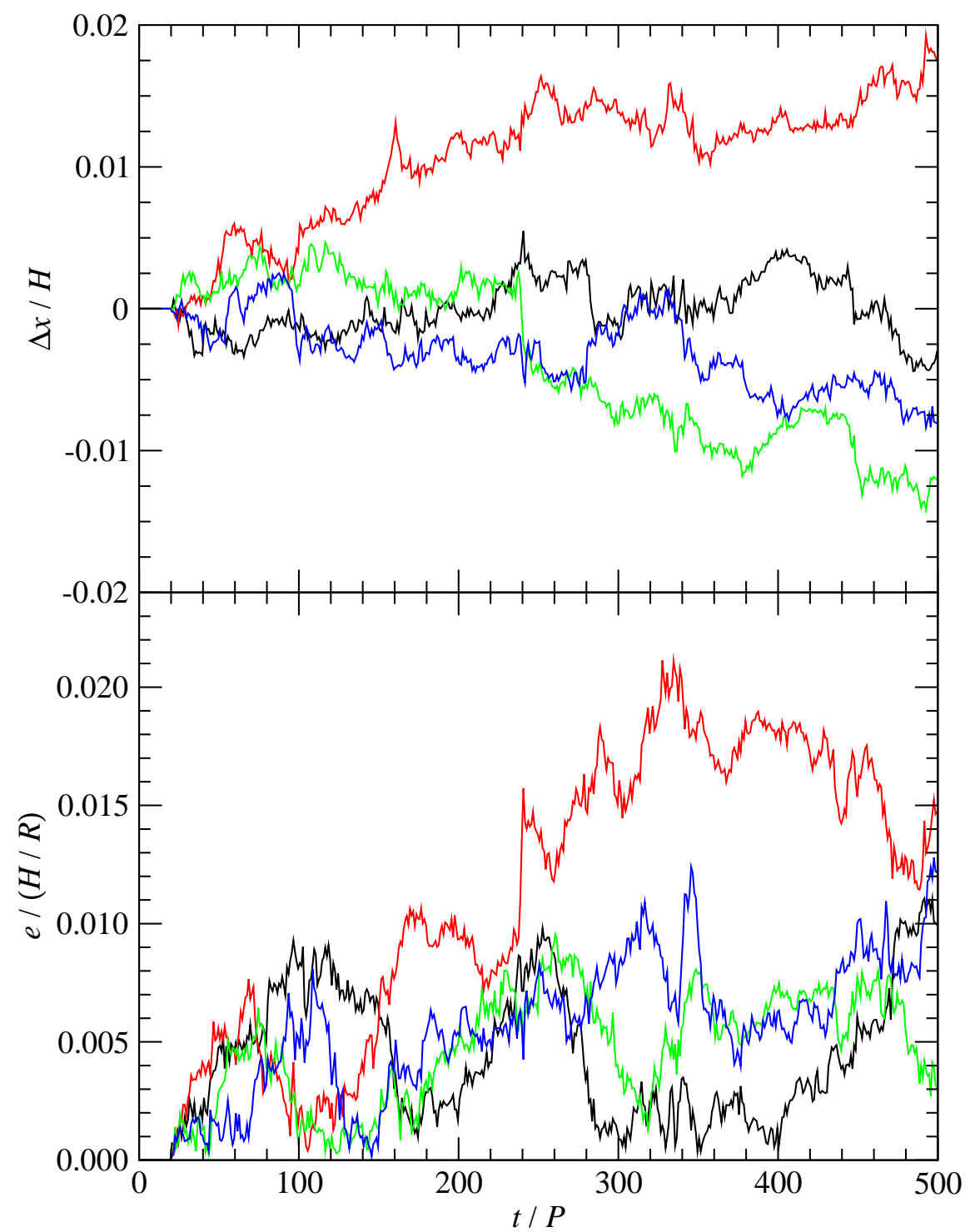

Fig. 4.- Time evolution of radial drift $\Delta x$ (top panel) and eccentricity $e$ (bottom panel) for four randomly selected particles with initial eccentricity $e_{0}=0$ moving in a low-mass disk. The eccentricity is in units of $H / R$, the ratio of one disk scale height to the distance of the shearing box to the host star.

\subsection{Radial Drift}

Histograms of the distribution of radial drifts at three different times are plotted in Figure 5 , The distribution of particles in radial drift resembles a normal distribution with its center located at approximately zero. This is not surprising since there is no preferred direction locally for the 


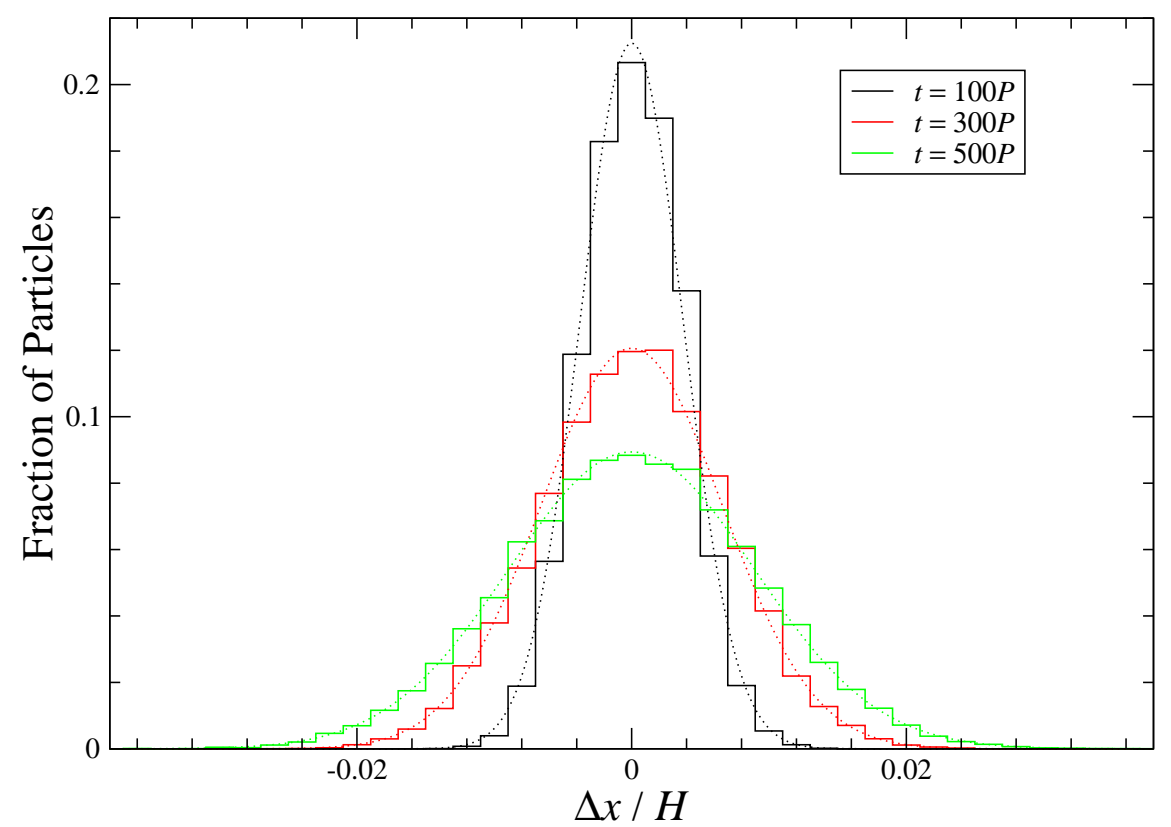

Fig. 5.- Distribution of particle radial drifts $\Delta x$ at three different times. These particles have initial eccentricity $e_{0}=0$ and evolve in a low-mass disk. The dotted lines are best fits in the form of normal distributions.

turbulence to generate a net torque. More interestingly, the width of the distribution increases with time. Although the hydromagnetic turbulence has no net effect on the orbital radius of the particles, it becomes more and more likely for any single particle to drift away from its original orbit as time increases. This could help a subset of particles to survive type-I migration, as suggested by JGM06 and Adams \& Bloch (2009).

Figure 66 shows the standard deviation of radial drift $\sigma(\Delta x)$ as a function of elapsed time $\Delta t \equiv t-t_{0}$ for particles of different initial eccentricity $e_{0}$ moving in different disk models. It steadily increases with time, with little difference between particles of different initial eccentricities. Power-law fitting results in time indices of about $0.52-0.58$, just slightly larger than $1 / 2$. This is consistent with the proposition that the "random walk" of the particles caused by hydromagnetic turbulence and the resulting orbital evolution can be described as a diffusion process (JGM06; OIM07; Rein \& Papaloizou 2009). We also find the dependence of orbital properties on the disk gravity, as measured by the dimensionless parameter $\xi$, by comparing the results from our low-mass and high-mass disk models (see \$2.3). As demonstrated in Figure 6, radial drifts in these models roughly coincide after being scaled with $\xi$, indicating a dependence close to linear. Our best fit to the results shown in Figure 6 is

$$
\sigma(\Delta x)=(3.8 \pm 0.4) \times 10^{-4} \xi H\left(\frac{\Delta t}{P}\right)^{1 / 2} .
$$




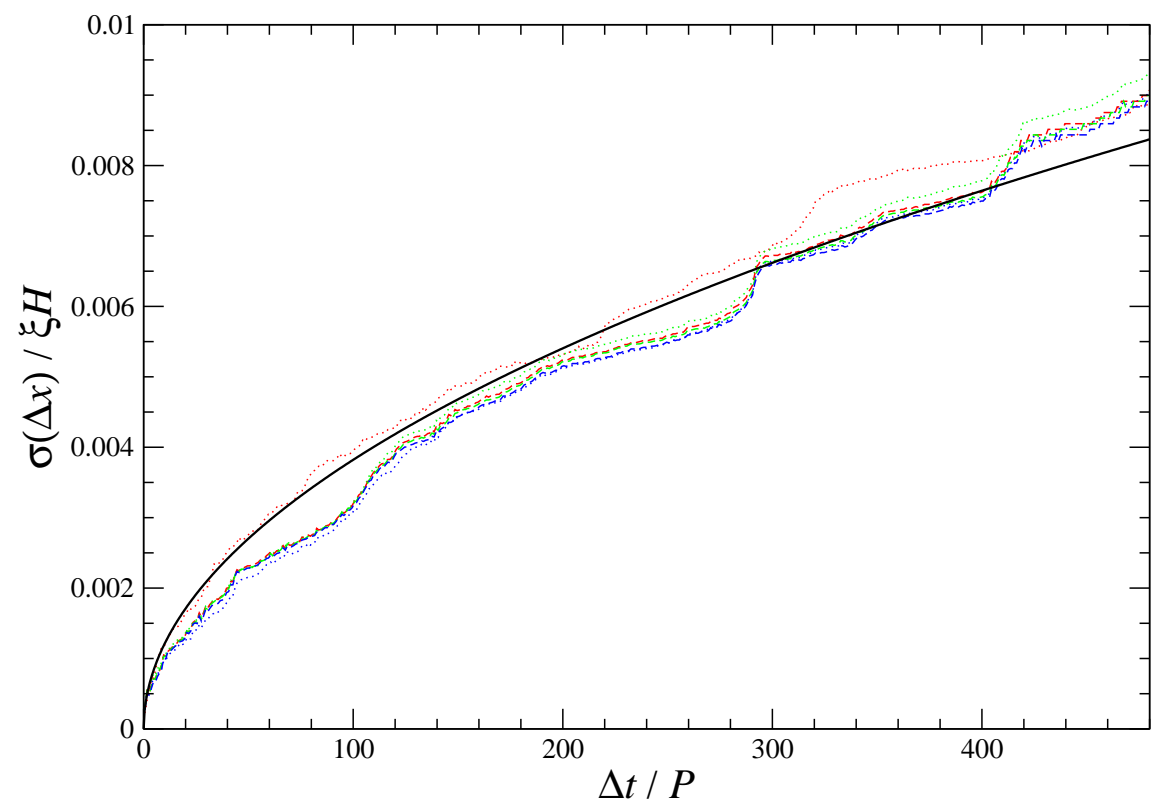

Fig. 6.- Standard deviation of radial drift $\sigma(\Delta x)$ as a function of elapsed time $\Delta t$. The dotted lines are obtained from low-mass disks $(\xi=1)$ while the dashed lines are from high-mass disks $(\xi=4 \pi)$. Particles with initial eccentricities $e_{0}=0,0.1(H / R)$, and $0.2(H / R)$ are denoted by red, green, and blue lines, respectively. The black solid line is the best fit to all six curves.

Using a heuristic, stochastic torque formula for hydromagnetic turbulence derived by LSA04 based on global disk models, OIM07 conducted orbital integrations of particles. The disk models they have studied are about 10-100 times less massive than the MMSN, but they have reported their scaling with varying disk mass. Note that the absence of an $m=1$ mode was assumed in OIM07, where the integer $m$ represents the Fourier decomposition of density structure in azimuthal angle (instead of spiral mode as in density wave theories). By extrapolating their results to values appropriate for our low-mass disk model with $\xi=1$, and considering their fiducial magnitudes for the stochastic torques, we find that our measured spread of radial drift (eq. [14]) is roughly consistent with theirs at 1 AU.

\subsection{Eccentricity}

Figure $7 \mathrm{a}$ shows the histograms of eccentricity at three different times for particles with initial eccentricity $e_{0}=0$ moving in the low-mass disk model. The distributions appear close to a Rayleigh 

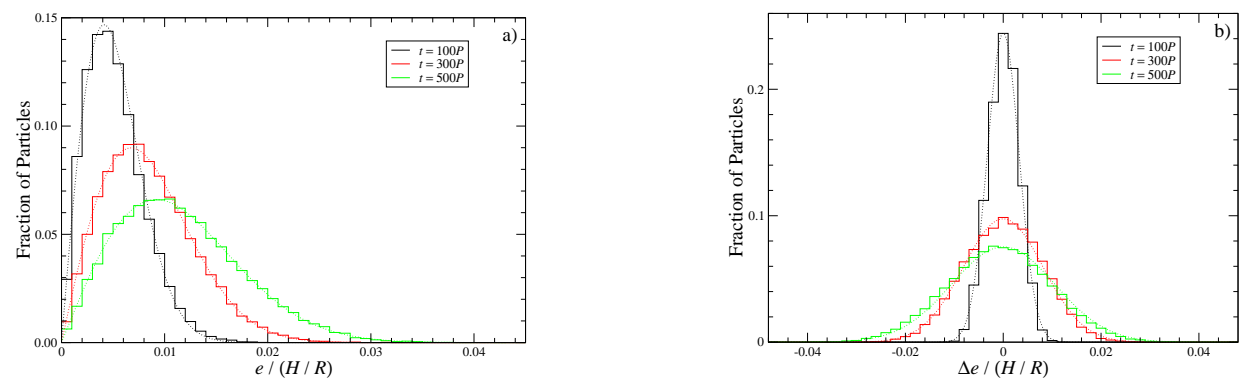

Fig. 7.- Distribution of particles in (a) eccentricity $e$ for particles with initial eccentricity $e_{0}=0$ and (b) eccentricity deviation $\Delta e=e-e_{0}$ for particles with $e_{0}=0.1(H / R)$ at three different times. These particles move in a low-mass disk. The dotted lines are best fits in the form of (a) Rayleigh and (b) normal distributions.

distribution:

$$
f(e)=\left(\frac{e}{\sigma^{2}}\right) \exp \left(-\frac{e^{2}}{2 \sigma^{2}}\right)
$$

where $\sigma$ is a constant proportional to both the peak and the width of the distribution. The standard deviation of this distribution is given by $\sigma \sqrt{(4-\pi) / 2}$. As shown in Figure 7 a, both the eccentricity at the peak value and the width of the distribution increase with time, so it appears that eccentricity is excited by hydromagnetic turbulence. This eccentricity growth has also been reported by N05 and OIM07. We remark that the mean eccentricity of particles with $e_{0}=0$ at $t=500 P$ is about $0.012(H / R)$ and $0.16(H / R)$ for the low-mass and the high-mass disk models, respectively, which correspond to epicycle motions covering about 1.5 and 20 grid zones in radial direction.

However, a different distribution obtains for particles with nonzero initial eccentricity. Figure $7 \mathrm{~b}$ shows histograms of eccentricity deviation $\Delta e=e-e_{0}$ at three different times for particles of initial eccentricity $e_{0}=0.1(H / R)$. They are similar to a normal distribution with a constant mean, implying that the average eccentricity of these particles remains constant at the initial eccentricity (see also OIM07). The width of the distribution does increase with time. Therefore, hydromagnetic turbulence does not necessarily excite the eccentricity of particles; it can also act to damp existing eccentricity of some particles. The distribution found for particles with $e_{0}=0$ is just a special case of this general behavior: since eccentricity is a positive definite quantity and the initial eccentricity is zero, it is no surprise that a normal distribution for the eccentricity deviation, $f(\Delta e)=\exp \left(-\Delta e^{2} / 2 \sigma^{2}\right) / \sqrt{2 \pi} \sigma$, manifests as a Rayleigh distribution for the eccentricity itself (eq. [15]).

Figure 8 shows the standard deviation of eccentricity deviation $\sigma(\Delta e)$ as a function of elapsed time $\Delta t$. Note that to be consistent with a normal distribution as discussed above, we have multiplied the standard deviation measured for particles with $e_{0}=0$ by a factor of $\sqrt{2 /(4-\pi)}$. As in the case of radial drift found in 93.4 , little difference exists between particles with different initial eccentricities and the results scale linearly with the dimensionless parameter $\xi$, which measures the 


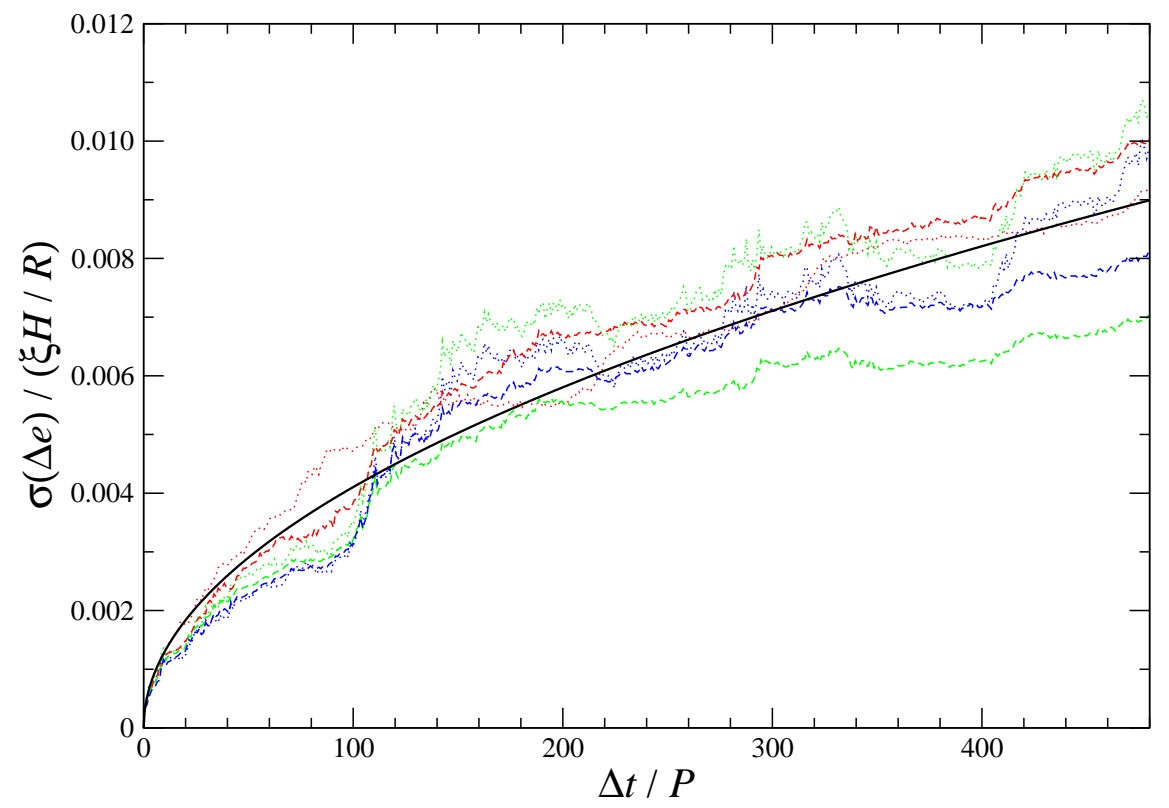

Fig. 8.- Standard deviation of eccentricity deviation $\sigma(\Delta e)$ as a function of elapsed time $\Delta t$. The line styles and colors are the same as in Figure 6. The standard deviation measured for particles with initial eccentricity $e_{0}=0$ has been multiplied by a factor of $\sqrt{2 /(4-\pi)}$ to be consistent with a normal distribution (see $\$ 3.5)$.

strength of the disk gravity. Chi-square fitting with a $t^{1 / 2}$ dependence to all six models leads to (Fig. 8)

$$
\sigma(\Delta e)=(4.1 \pm 0.6) \times 10^{-4} \xi\left(\frac{H}{R}\right)\left(\frac{\Delta t}{P}\right)^{1 / 2} .
$$

Note that at $1 \mathrm{AU}$ in an MMSN disk, $\xi \simeq 1, H / R \simeq 0.07$, and $P=1 \mathrm{yr}$, and thus the eccentricity deviation due to hydromagnetic turbulence only amounts to about 0.03 in $1 \mathrm{Myr}$.

As with the case of radial drift discussed in $\$ 3.4$, our measured spread of eccentricity is in approximate agreement with OIM07. On the other hand, N05 has reported a typical eccentricity growth of $e \simeq 0.03$ for $\Delta t \sim 100 P$. With $\xi \simeq 6.6$ at radius $r=2.7$ in the model reported by N05 (R. P. Nelson 2009, private communications) and $H / R=0.07$, the eccentricity deviation given by equation (16) is about one order of magnitude less than what is reported by N05. For a fair comparison, we have also run a model with similar conditions to those in a local region at $r=2.7$ of N05. In this model, the computational domain is $(2.56,4.74,1.08) H$ with a $32^{3}$ grid such that the resolution is approximately the same as in N05. Furthermore, the external field is set at $B_{\text {ext }}=0.04$ $\left(\beta_{\text {ext }}=2.5 \times 10^{4}\right)$, which leads to $\langle\alpha\rangle \sim 0.007$ and $\langle 1 / \beta\rangle \sim 0.029$ at saturation stage. Nevertheless, the resulting orbital properties of zero-mass particles do not show significant changes from what are given by equations (14) and (16). 
This inconsistency might be attributed to the difference between global and local disk models. OIM07 have reported that the inclusion of an $m=1$ mode in their torque formula induces a 10-100 times greater impact of hydromagnetic turbulence on particle orbits, using the calibration with global MHD simulations provided by LSA04. By inspection of LSA04 as well as N05, the density structures in their global models often extend more than $\pi / 2$ in azimuthal angle, leading to dominant amplitude of the $m=1$ mode. On the other hand, using local models with mean azimuthal fields, Guan et al. (2009) have reported that the coherent structures induced by the MRI are localized with a correlation length of about $(0.05,0.32,0.05) H$. Therefore, it is possible that current global models might not have enough resolution to model such fine structures, which were then spuriously connected into extended features. To validate this conjecture, though, global disk models capable of resolving localized structures are needed.

On the other hand, there are at least two ways in which our local models could underestimate the influence of the MRI turbulence. Firstly, increasing the box size of a local model beyond $2 H$ can amplify the turbulent viscosity $\alpha$ by as much as a factor of two (Johansen et al. 2009). However, Figure 2 suggests that the magnitude of density perturbations is only weakly sensitive to the strength of the $\alpha$ viscosity. Power-law regression analysis of Figure 2 leads to $\Delta \rho / \rho_{0} \propto \beta_{\text {ext }}^{-1 / 2}$ and $\alpha \propto \beta_{\text {ext }}^{-3 / 4}$, and thus $\Delta \rho \propto \alpha^{2 / 3}$. A factor of two increase in $\alpha$ thus corresponds to about a factor of 1.6 increase in density perturbation, which seems not sufficient to explain the discrepancy between global and local models. Secondly, the absence of higher-order curvature terms in the local shearing box approximation offers another plausible explanation for the discrepancy.

\subsection{Horizontal Velocity Dispersion}

Another important quantity in the study of planetesimal dynamics is the velocity dispersion of the particles. We calculate the radial and the azimuthal components of velocity dispersion by taking the standard deviations of radial and azimuthal velocities for all particles, i.e., $\sigma\left(u_{x}\right)$ and $\sigma\left(u_{y}\right)$, respectively. Since all the dynamical equations are linearized in terms of $x / R$ in the local shearing box approximation, the background shear flow is uniform irrespective of position, so the velocity dispersion for all particles in the computational domain is a well-defined local quantity. Figure 9 plots $\sigma\left(u_{x}\right)$ and $\sigma\left(u_{y}\right)$ measured for particles with zero initial eccentricity in a low-mass disk as a function of time $t$. The velocity dispersion monotonically increases with time, and thus hydromagnetic turbulence tends to steadily heat up a planetesimal disk. Note that $\sigma\left(u_{y}\right) \sim \sigma\left(u_{x}\right) / 2$, a sanity check that our results are consistent with a swarm of non-interacting particles moving epicyclically in a Keplarian disk.

Figure 10 compiles the radial velocity dispersions $\sigma\left(u_{x}\right)$ as a function of elapsed time $\Delta t$ measured for particles with different initial eccentricity $e_{0}$ moving in disks of different disk gravity. As is the case with the radial drift and eccentricity deviation discussed in 33.4 and 33.5 , the results depend linearly on $\xi$, the strength of disk gravity. On the other hand, there seems to be a slightly enhanced effect for particles with $e_{0}>0$. Given the uncertainty involved in the numerical 


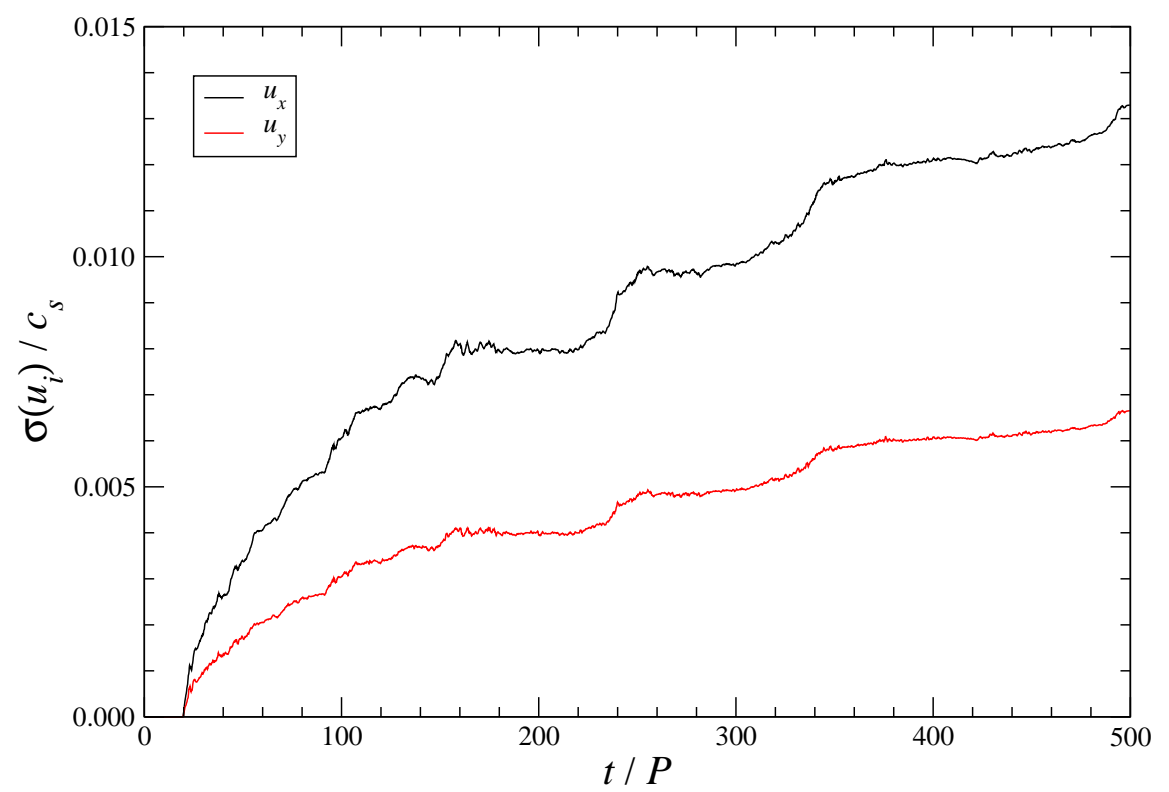

Fig. 9.- Radial and azimuthal components of velocity dispersion $\sigma\left(u_{x}\right)$ and $\sigma\left(u_{y}\right)$ as a function of time $t$ for particles with initial eccentricity $e_{0}=0$ moving in a low-mass disk. They are presented as running averages over one orbital period $P$.

simulations, we assume it is a secondary effect as a first approximation. The best fit to all six models is then given by

$$
\sigma\left(u_{x}\right)=(7.6 \pm 1.6) \times 10^{-4} \xi c_{s}\left(\frac{\Delta t}{P}\right)^{1 / 2}
$$

The corresponding timescale $\tau_{T}$ for turbulent excitation of velocity dispersion can be estimated by $\tau \equiv \sigma /(\mathrm{d} \sigma / \mathrm{d} \Delta t)$, and we find from equation (17)

$$
\tau_{T}=\left(3.4 \times 10^{6} P\right) \xi^{-2}\left[\frac{\sigma\left(u_{x}\right)}{c_{s}}\right]^{2} .
$$

As noted in 93.5 , hydromagnetic turbulence can act either to excite the eccentricities of planetesimals or to circularize their finite eccentricity orbits. However, a perfectly cold disk of planetesimals with nonzero initial eccentricity but vanishing velocity dispersion will be monotonically heated by the turbulence. The velocity dispersion increases with time while the mean eccentricity may remain unchanged unless a significant fraction of particles are circularized. Therefore, the eccentricity may not necessarily be proportional to the velocity dispersion for a swarm of planetesimals moving through hydromagnetic turbulence.

The velocity dispersion of a planetesimal disk also grows with time due to mutual gravitational scattering. The corresponding timescale for a swarm of identical particles of mass $m_{p}$ can be 


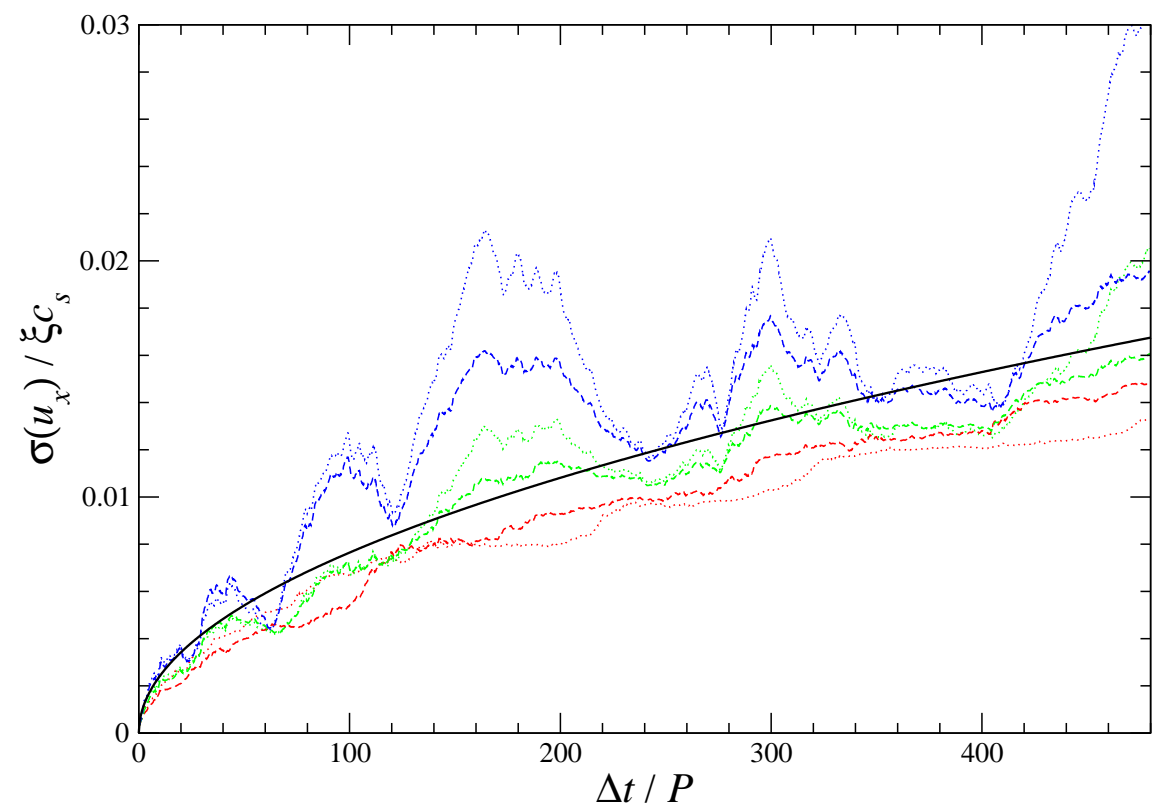

Fig. 10.- Radial component of velocity dispersion $\sigma\left(u_{x}\right)$ as a function of elapsed time $\Delta t$. It is presented as running averages over one orbital period $P$. The dotted lines are obtained from low-mass disks $(\xi=1)$ while the dashed lines are from high-mass disks $(\xi=4 \pi)$. Particles with initial eccentricities $e_{0}=0,0.1(H / R)$, and $0.2(H / R)$ are denoted by red, green, and blue lines, respectively. The solid black line is the best fit to all six curves, assuming no explicit dependence on $e_{0}$.

estimated as (e.g., Papaloizou \& Terquem 2006)

$$
\tau_{G S}=\frac{\sigma^{3}\left(u_{x}\right)}{8 \sqrt{\pi} G^{2} m_{p}^{2} n_{p} \ln \Lambda_{p}}\left[\frac{\sqrt{3}}{4} \ln \left(\frac{2+\sqrt{3}}{2-\sqrt{3}}\right)-1\right]^{-1},
$$

where $n_{p}$ is the number density of planetesimals and $\Lambda_{p}=3 \sigma^{2}\left(u_{x}\right) H_{p} / 4 G m_{p}$, in which $H_{p} \sim$ $\sqrt{2} \sigma\left(u_{z}\right) / \Omega_{K}$ is the scale height of the planetesimal disk determined by the vertical velocity dispersion $\sigma\left(u_{z}\right)$. To find $\tau_{G S}$ in physical units, we assume for simplicity that most of the solid material in a protoplanetary disk is concentrated in planetesimals, and thus $n_{p} m_{p} \sim \varepsilon \rho_{0} c_{s} / \sigma\left(u_{z}\right)$ where $\varepsilon$ is the solid-to-gas ratio. We also assume $\sigma\left(u_{z}\right) \sim \sigma\left(u_{x}\right) / 2$, so that equation (19) becomes

$$
\tau_{G S} \simeq \frac{\sqrt{\pi} P^{2} \sigma^{4}\left(u_{x}\right)}{4 \varepsilon \xi c_{s} G m_{p} \ln \Lambda_{p}}\left[\frac{\sqrt{3}}{4} \ln \left(\frac{2+\sqrt{3}}{2-\sqrt{3}}\right)-1\right]^{-1},
$$

with

$$
\Lambda_{p} \simeq \frac{3 \sqrt{2} P \sigma^{3}\left(u_{x}\right)}{16 \pi G m_{p}}
$$


We further focus our discussion on a velocity scale of order $v_{\text {esc }}$, the escape velocity at the surface of a planetesimal:

$$
v_{\mathrm{esc}}=\left(\frac{32 \pi}{3} G^{3} m_{p}^{2} \rho_{p}\right)^{1 / 6},
$$

where $\rho_{p}$ is the material density of the planetesimal. This scale is of critical interest for planetary cores to accrete solid material; particles with relative velocities of order $v_{\text {esc }}$ are more likely to coalesce into larger bodies than to be eroded into smaller pieces. By assuming $\sigma\left(u_{x}\right) \sim v_{\text {esc }}$, the timescales for heating a planetesimal disk by hydromagnetic turbulence and gravitational scattering become

$$
\tau_{T}=\left(1.1 \times 10^{2} \mathrm{yr}\right) \xi^{-2}\left(\frac{\rho_{p}}{3 \mathrm{~g} \mathrm{~cm}^{-3}}\right)^{1 / 3}\left(\frac{m_{p}}{10^{18} \mathrm{~g}}\right)^{2 / 3}\left(\frac{c_{s}}{10^{5} \mathrm{~cm} \mathrm{~s}^{-1}}\right)^{-2}\left(\frac{P}{\mathrm{yr}}\right)
$$

and

$$
\tau_{G S} \simeq\left(\frac{1.4 \times 10^{5}}{\ln \Lambda_{p}} \mathrm{yr}\right) \xi^{-1}\left(\frac{\varepsilon}{0.01}\right)^{-1}\left(\frac{\rho_{p}}{3 \mathrm{~g} \mathrm{~cm}^{-3}}\right)^{2 / 3}\left(\frac{m_{p}}{10^{18} \mathrm{~g}}\right)^{1 / 3}\left(\frac{c_{s}}{10^{5} \mathrm{~cm} \mathrm{~s}^{-1}}\right)^{-1}\left(\frac{P}{\mathrm{yr}}\right)^{2}
$$

with

$$
\Lambda_{p} \simeq 6.9 \times 10^{3}\left(\frac{\rho_{p}}{3 \mathrm{~g} \mathrm{~cm}^{-3}}\right)^{1 / 2}\left(\frac{P}{\mathrm{yr}}\right)
$$

respectively.

With the scales assumed above and at $1 \mathrm{AU}$ in an MMSN disk, $\tau_{G S} \sim 2 \times 10^{4}$ yr while $\tau_{T} \sim 100 \mathrm{yr}$; for $m_{p}=0.001 M_{\oplus}, \tau_{G S} \sim 3 \times 10^{6} \mathrm{yr}$ and $\tau_{T} \sim 4 \times 10^{6} \mathrm{yr}$. Therefore, hydromagnetic turbulence probably dominates the heating of a disk of kilometer-sized planetesimals, while gravitational scattering may be more important for objects approaching Earth size. Note that $\tau_{G S}$ increases more steeply with $\sigma\left(u_{x}\right)$ and $P$ but decreases less rapidly with $\xi$ than $\tau_{T}$ (eqs. 18, and [20]), and thus hydromagnetic turbulence probably gains dominance over gravitational scattering for larger velocity dispersion, larger distance to the host star, and/or more massive gas disks than assumed here.

\section{IMPLICATIONS FOR PLANET FORMATION}

In this section, we apply our results on the orbital evolution of zero-mass particles to two specific problems in planet formation. First, we estimate the strength of diffusive migration of protoplanets due to hydromagnetic turbulence and discuss their survivability under type-I migration following the analytical framework established by JGM06. Secondly, we revisit the proposition of IGM08 that planetesimals may suffer from collisional destruction as a result of hydromagnetic turbulence excitation of velocity dispersion among them. 


\subsection{Diffusive Migration of Protoplanets}

Using a Fokker-Planck formalism, JGM06 derived an advection-diffusion equation to describe the evolution of the distribution of protoplanets under the influence of both type-I migration and hydromagnetic turbulence. Adams \& Bloch (2009) have further consolidated the analysis by studying more realistic disk density structure with both spatial and time dependence. These authors have found that turbulence tends to reduce the lifetimes of most protoplanets while allowing some of them to linger long enough to survive rapid inward type-I migration. The likelihood of producing a planetary system with a specific configuration sensitively depends on the strength of the diffusive migration induced by the turbulence, however. JGM06 and Adams \& Bloch (2009) calibrated the turbulence strength with the global disk models computed by LSA04, Nelson \& Papaloizou (2004), and N05. In contrast, using a local disk model, OMM07 found that the strength might be several orders of magnitude less than what was estimated by JGM06. In this section, we provide a new assessment based on the disk models studied in this work.

As shown in 93.4 , an initial delta function in the distribution of mean orbital radii of zero-mass particles is spread with time into a normal distribution of constant mean. A $t^{1 / 2}$ time dependence for the standard deviation of the distribution suggests that this process can be described by a diffusion equation of the form

$$
\frac{\partial f}{\partial t}=\frac{\partial}{\partial x}\left(\mathcal{D} \frac{\partial f}{\partial x}\right)
$$

where $\mathcal{D}$ is the diffusion coefficient and $f=f(t, x)$ is the distribution function: $f(t, x) \mathrm{d} x$ is the probability of finding a particle with a radial displacement in $(x, x+\mathrm{d} x)$ at time $t$. Let us write,

$$
\begin{gathered}
f(x, t)=\frac{1}{\sigma(t) \sqrt{2 \pi}} \exp \left[-\frac{x^{2}}{\sigma^{2}(t)}\right], \\
\sigma(t)=\sigma_{1}\left(\frac{t}{P}\right)^{1 / 2} \quad(t>0),
\end{gathered}
$$

where $\sigma_{1}$ is a proportionality constant. Substituting equation (27) into equation (26), we find that $\mathrm{d} \sigma / \mathrm{d} t=2 \mathcal{D} / \sigma$ and thus with $\sigma\left(t \rightarrow 0^{+}\right)=0$,

$$
\sigma(t)=2 \mathcal{D}^{1 / 2} t^{1 / 2}
$$

By comparing equations (28) and (29), the diffusion coefficient $\mathcal{D}$ is related to $\sigma_{1}$ and $P$ by

$$
\mathcal{D}=\frac{\sigma_{1}^{2}}{4 P}
$$

Our fit to the simulated results, given by Equation (14), can be substituted here to find

$$
\mathcal{D}(R)=3.6 \times 10^{-8} \xi^{2}\left(\frac{H^{2}}{P}\right)
$$


by identifying $t$ with $\Delta t$. In this derivation, we have assumed that the stochastic torque exerted by hydromagnetic turbulence is a local process such that the diffusion coefficient $\mathcal{D}$ is sufficiently constant near $x=0$.

We are now in a position to estimate the diffusion coefficient $D(J)$ of JGM06 in comparision to $\mathcal{D}(R)$, where $J=m_{p}\left(G M_{\star} R\right)^{1 / 2}$ is the orbital angular momentum of a protoplanet of mass $m_{p}$ orbiting a star of mass $M_{\star}$ on a quasi-circular orbit at a radial distance $R$. Since $D(J)$ and $\mathcal{D}(R)$ have dimensions of $\left[J^{2} / t\right]$ and $\left[R^{2} / t\right]$, respectively, they may be related by $D(J) \sim \mathcal{D}(R)(\partial J / \partial R)^{2}=$ $(J / 2 R)^{2} \mathcal{D}(R)$. Using dimensional arguments, JGM06 have defined a dimensionless parameter $\epsilon$ to describe the uncertainties associated with hydromagnetic turbulence:

$$
D(J)=\left(2.1 \times 10^{-3}\right) \epsilon(2 \pi)^{3} \frac{\Sigma^{2} J^{7}}{G^{2} M_{\star}^{4} m_{p}^{5}}=\frac{\left(2.1 \times 10^{-3}\right)}{16 \pi} \epsilon \xi^{2}\left(\frac{H}{R}\right)^{2}\left(\frac{J^{2}}{P}\right) .
$$

By comparing equations (31) and (32), we find that $\epsilon \simeq 2.2 \times 10^{-4}$. Note that $\epsilon$ is a constant independent of $R$ given our scalings, in agreement with the assumption of constant $\epsilon$ made by JGM06.

Our estimated value of $\epsilon$ is about one order of magnitude smaller than what was reported by OMM07 for the case of stratified disks in the limit of ideal MHD. To identify the reason for this discrepancy, we further evaluate the magnitude and the correlation time of the torques exerted by the turbulent gas on the particles. Firstly, the root-mean-square of the $y$-component of the gravitational force per unit mass exerted by the gas over all time and particles is $a_{y, \mathrm{rms}}=(3.7 \times$ $\left.10^{-3}\right) \xi H P^{-2}=\left(4.2 \times 10^{-3}\right) \times(2 \pi G \Sigma)$. We find negligible difference between torques calculated following the particles along their orbits and those calculated at the fixed center of the box. The magnitude we have obtained is reasonably consistent with the value of $a_{y, \text { rms }}=\left(3.2 \times 10^{-3}\right) \times$ $(2 \pi G \Sigma)$ reported by OMM07 at the same numerical resolution. Secondly, we plot in Figure 11 the autocorrelation functions (ACFs) of $a_{y}$ for several randomly selected particles as well as at the center of the box. The results are again similar to what was reported by OMM07, indicating a similar estimate for the correlation time, $\tau_{c}$. The source of the discrepancy appears to be neither of these factors.

We notice, though, that OMM07 could have overestimated the correlation time by taking the second zero-crossing of the ACF. Strictly speaking, the correlation time should instead be computed by integrating an ensemble average of the ACF over all possible realizations, as motivated by the definitions of the diffusion coefficient and the correlation time in JGM06:

$$
D(J) \equiv \frac{1}{2} \int_{-\infty}^{\infty} \overline{\delta \Gamma\left(t-\frac{\tau}{2}, J\right) \delta \Gamma\left(t+\frac{\tau}{2}, J\right)} \mathrm{d} \tau
$$

and

$$
\tau_{c} \equiv D(J) / \overline{\delta \Gamma^{2}(t, J)}
$$

where $\delta \Gamma(t, J)$ is the fluctuating part of the torque and the overline denotes ensemble average. Since we distribute numerous particles uniformly over the entire computational domain, the ACF 


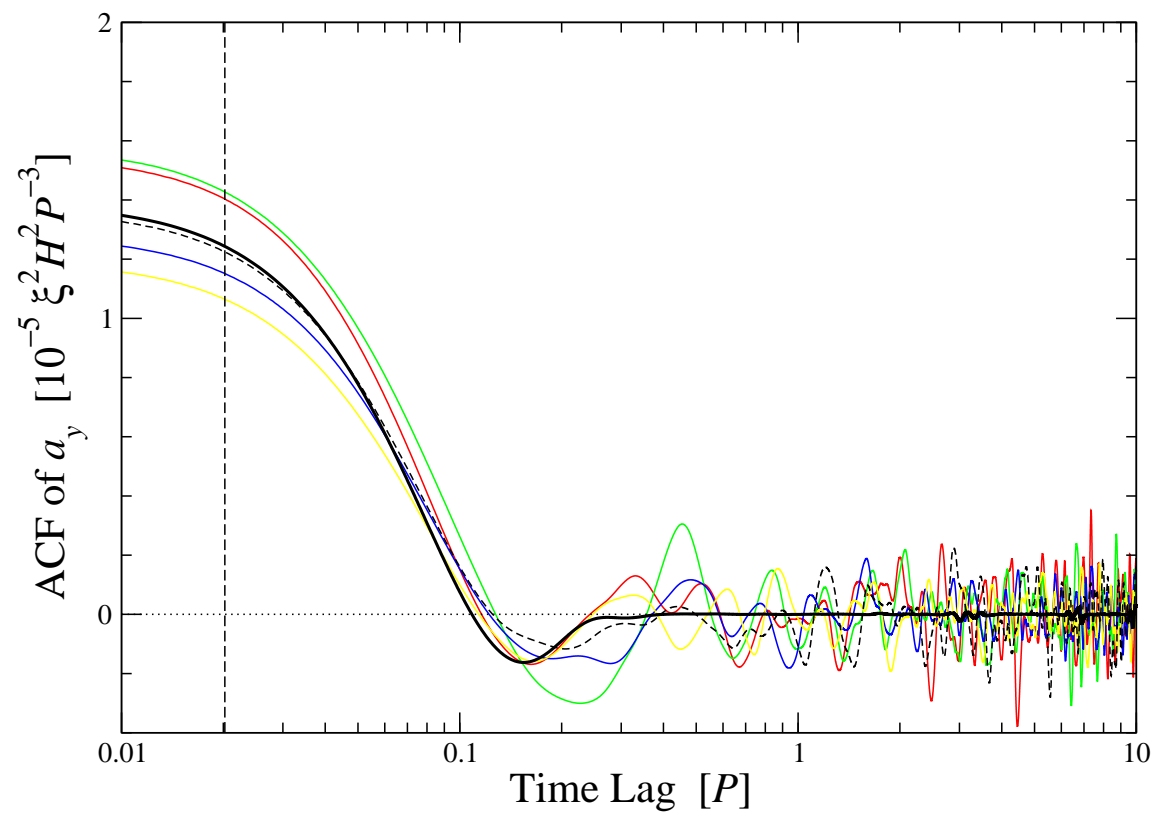

Fig. 11. - Autocorrelation functions of the azimuthal (torque) component of the gravitational force per unit mass, $a_{y}$, exerted by the turbulent gas. The short-dashed black curve corresponds to $a_{y}$ calculated at the center of the box, while the various colored curves correspond to $a_{y}$ calculated following the orbits of several randomly selected particles. The average over all particles is given by the solid black line, which shows almost no power beyond the second zero crossing. The vertical long-dashed black line indicates the estimated correlation time of the torques with equation (35).

obtained for each particle can be considered as one realization, and the average of the ACFs over all particles may resemble the true ensemble average. This averaged ACF is shown as the solid black curve in Figure 11. Note that the oscillation occurring at time lag longer than the second crossing for each particle is much reduced, indicating the noise nature of the autocorrelation at long time lag. However, the negative value of the ACF between the first two zero-crossings remains significant. This interval represents the anti-diffusion nature of the stochastic torques that we believe could be responsible for reducing the diffusion coefficient. Therefore, we suggest that a better approximation for the correlation time in accordance with equations (33) and (34) is

$$
\tau_{c} \approx \frac{\int_{0}^{\infty} \overline{\mathrm{ACF}(\tau)} \mathrm{d} \tau}{2 \overline{\mathrm{ACF}(0)}},
$$

where $\overline{\mathrm{ACF}(t)}$ is the ensemble-averaged $\mathrm{ACF}$ of the torque per unit mass $a_{y}$ as a function of time lag $t$. Using equation (35), we find that $\tau_{c}=0.020 P$, about one order of magnitude less than the value of $\tau_{c}=0.31 P$ reported by OMM07. With the same approximation $D(J) \simeq m_{p}^{2} R^{2} \overline{a_{y}^{2}} \tau_{c}$ used by $\mathrm{OMM} 07$, we find that $\epsilon \simeq 1.7 \times 10^{-4}$, in excellent agreement with the estimate of $\epsilon \simeq 2.2 \times 10^{-4}$ we derived from our direct measurement of particle radial drifts. 
According to Figures 6 and 7 of JGM06, the value of $\epsilon$ we have inferred from our simulations indicates that advective (type-I) migration dominates over diffusive (stochastic) migration for the parameter space JGM06 have investigated. For an Earth-mass protoplanet at a radial distance up to $100 \mathrm{AU}$ in the MMSN disk and in a viscous disk with $\alpha=0.02$, advection dominates when $\epsilon \lesssim 10^{-2}$ and $10^{-1}$, respectively. For a protoplanet of mass as low as $0.01 M_{\oplus}$ at $R=10 \mathrm{AU}$ in the same disk models, advection dominates when $\epsilon \lesssim 10^{-3}$ and $10^{-2}$, respectively. The critical distance and mass for the transition between dominance of advection and diffusion given our small estimate of $\epsilon$ is outside of the parameter space explored by JGM06. By inspection of Figure 7 in JGM06, though, the transition masses for an object at 10 AU in the MMSN disk and in the viscous disk probably lies at about $10^{-3}$ and $10^{-4} M_{\oplus}$, respectively. Therefore, our results suggest that hydromagnetic turbulence does not significantly affect the secular migration of Earth-sized protoplanets in regimes of current astrophysical interest. Nevertheless, torques exerted by turbulent density perturbations seem to be a dominant agent determining the orbital dynamics of kilometer-sized planetesimals.

\subsection{Collisional Destruction of Planetesimals}

IGM08 have suggested that hydromagnetic turbulence may inhibit the growth of kilometersized planetesimals. They argue that the velocity dispersion excited by the turbulence could be so large that collisions between planetesimals exceed their material strength or self-gravity, leading to destruction. Their conclusion, however, relies on orbital integrations incorporating the heuristic, stochastic formulas for the time history of gravitational torques provided by LSA04, which in turn were calibrated using MHD simulations of a global disk model. As pointed out in \$3.5, a major inconsistency exists between global and local models. Since the latter shows an order of magnitude lower effect on orbital dynamics of planetesimals, it is worthwhile revisiting the planetesimal growth problem in light of the results highlighted in this work.

For comparison purposes, we adopt the same scalable MMSN disk model as used by IGM08. The gas density and the speed of sound in the mid-plane are given by

$$
\rho_{0}=\left(1.8 \times 10^{-9} \mathrm{~g} \mathrm{~cm}^{-3}\right) f_{g}\left(\frac{R}{\mathrm{AU}}\right)^{-11 / 4}
$$

and

$$
c_{s}=\left(1.1 \times 10^{5} \mathrm{~cm} \mathrm{~s}^{-1}\right)\left(\frac{R}{\mathrm{AU}}\right)^{-1 / 4},
$$

respectively, where a solar-type host $\operatorname{star}\left(M_{\star}=M_{\odot}\right)$ is assumed and $f_{g}$ is a scale factor. When $f_{g}=1$, the disk mass is about 1.4 times that of an MMSN disk. The corresponding $\xi$-parameter and ratio of disk scale height to radial distance then become

$$
\xi=1.5 f_{g}\left(\frac{R}{\mathrm{AU}}\right)^{1 / 4}
$$


and

$$
\frac{H}{R}=0.052\left(\frac{R}{\mathrm{AU}}\right)^{1 / 4}
$$

respectively. Substituting equations (38) and (39) into equation (16), we arrive at

$$
\sigma(\Delta e)=3.2 \times 10^{-5} f_{g}\left(\frac{R}{\mathrm{AU}}\right)^{-1 / 4}\left(\frac{\Delta t}{\mathrm{yr}}\right)^{1 / 2} .
$$

Comparing equation (40) with equation (13) of IGM08 with the understanding that $\sigma(e)=\sigma(\Delta e) \sqrt{(4-\pi) / 2}$ for particles with zero initial eccentricity (see \$3.5), we find the value of the dimensionless parameter $\gamma$ - a measure of the strength of hydromagnetic turbulence used by IGM08 — in our orbital integrations to be $\gamma \simeq 2.1 \times 10^{-4}$. The rest of the analysis performed by IGM08 remains unchanged since the effects induced by hydromagnetic turbulence are all incorporated in the parameter $\gamma$.

The rather small value of $\gamma$ we have estimated indicates that hydromagnetic turbulence does not pose a serious threat to the growth of kilometer-sized planetesimals. IGM08 have compared the critical radii of planetesimals for accretive and erosive regimes due to different turbulence strengths $\gamma=10^{-2}, 10^{-3}$, and $10^{-4}$ in their figures 3 , 4, and 5 , respectively. Therefore, the value of $\gamma$ we have measured points to a scenario closer to what is predicted by Figure 5 of IGM08. In this scenario, the erosive regimes only appear in the outer regions ( $\gtrsim 30 \mathrm{AU}$ ) of a young protoplanetary disk and disappear rapidly with decreasing disk mass. Kilometer-sized planetesimals safely evade collisional destruction in the inner regions of the disk. We reiterate that the value of $\gamma$ measured here pertains to a local region with turbulent stresses such that $\alpha \sim 10^{-2}$ and $\Delta \rho / \rho_{0} \sim 10 \%$. As discussed in 93.5 , the validity of our results in a global context remains to be demonstrated by numerical experiments on a global disk model with a resolution capable of resolving the characteristic scales of coherent turbulence structures.

\section{CONCLUSIONS}

In this work, we have used local-shearing-box simulations to study the dynamics of massless planetesimals or protoplanets in a turbulent, isothermal, unstratified gas disk driven by the MRI. With a uniform, vertical magnetic field but without explicit physical dissipation, the saturated turbulence is maintained at a roughly constant level, showing convergence with increasing resolution. By adopting a suitable magnitude for the net magnetic flux, we produce a fiducial disk model with turbulent accretion at the level of $\alpha \sim 10^{-2}$ and with root-mean-square density perturbations $\Delta \rho / \rho_{0} \sim 10 \%$. As discussed in $₫ 2.3$, this model can be scaled to other physical systems of interest, as long as the assumption of negligible self-gravity of the gas remains valid. After the hydromagnetic turbulence in our fiducial model reaches saturation, we distribute numerous particles of zero mass and integrate their orbital motion under the gravitational influence of the turbulent gas.

The stochastic nature of the orbital evolution of these particles is evident, so we characterize

their orbital dynamics with statistical distributions, finding three major results. First, although the 
mean orbital radius does not change, particles slowly drift away from their original radius, so that the distribution of radii grows with time. Second, torques from density perturbations produced in hydromagnetic turbulence can both excite and damp the eccentricities of particle orbits, with again no change in the mean value, but a growing width of distribution if the particles possess non-negligible initial eccentricities. Finally, the planetesimal disk is heated up by the turbulence, a process dominating over gravitational scattering between particles in most physical conditions relevant to protoplanetary gas disks and planetesimal sizes. A corollary of these results is that eccentricity does not serve as a good indicator of the velocity dispersion of the particles.

The amplitude of orbital changes driven by the turbulence in our local models is about an order of magnitude less than what was reported in recent global models (LSA04; N05; IGM08). Two possible explanations for this discrepancy suggest themselves: either insufficient resolution in the global models or the lack of higher-order curvature terms in our local shearing box model, as discussed in 3.5 . If our local results are valid, they indicate that although hydromagnetic turbulence can drive radial diffusion, eccentricity variations, and relative velocities of planetesimals and protoplanets, these effects may not be dominant in determining their evolution. In particular, it appears that type-I migration dominates over turbulent radial drift for objects well above $10^{-4} \mathrm{M}_{\oplus}$. In addition, hydromagnetic turbulence might not be exciting sufficient velocity dispersion to drive planetesimals into an erosive regime that would inhibit their further growth. Before these results can be considered as robust, however, it will be necessary to elucidate, and hopefully reconcile, the differences that have appeared between global and local models.

We thank Jeffrey S. Oishi and Richard P. Nelson for the clarification of their works and useful discussions. The computations reported here were performed on the Columbia and Pleiades systems of the NASA Advanced Supercomputing (NAS) Division. This research is supported by the NASA Origins of Solar Systems Program under grant NNX07AI74G.

\section{A. VELOCITY OF A PARTICLE AT THE APOGEE OF ITS ORBIT}

In this section, we (re-)derive the velocity of a particle at the apogee of its elliptical orbit in the local shearing box approximation of a Keplarian disk. We repeat the equation of motion (8b) for a single particle without the gravity of the gas here:

$$
\begin{aligned}
\frac{\mathrm{d} u_{x}}{\mathrm{~d} t} & =2 \Omega_{K} u_{y} \\
\frac{\mathrm{d} u_{y}}{\mathrm{~d} t} & =-\frac{1}{2} \Omega_{K} u_{x},
\end{aligned}
$$

where we have dropped the subscript $p$ for clarity. Eliminating $u_{y}$ in equations (A1) and (A2) leads to

$$
\frac{\mathrm{d}^{2} u_{x}}{\mathrm{~d} t^{2}}+\Omega_{K}^{2} u_{x}=0 .
$$


By assuming the particle is at the apogee at $t=0$, the solution for $u_{x}$ is

$$
u_{x}=-A \sin \Omega_{K} t
$$

where $A$ is the amplitude of the radial velocity. Since $\mathrm{d} x / \mathrm{d} t=u_{x}$ (eq. [8a $)$, the radial oscillation is then

$$
\Delta x \equiv x-x_{0}=\frac{A}{\Omega_{K}} \cos \Omega_{K} t,
$$

where $x_{0}$ is the radial position of the center of the orbit. From equation (A2), the corresponding azimuthal velocity relative to the background shear flow is

$$
u_{y}=-\frac{1}{2} A \cos \Omega_{K} t=-\frac{1}{2} \Omega_{K} \Delta x
$$

Since the eccentricity $e$ of the orbit is related with the amplitude of the radial oscillation by $e \approx \Delta x(t=0) / R$, where $R$ is the distance to the central object,

$$
u_{y}(t=0)=-\frac{1}{2} R \Omega_{K} e=-\frac{1}{2} H \Omega_{K}\left(\frac{e}{H / R}\right),
$$

where $H$ is the disk scale height. This is just the initial condition (12) we set out to prove. Note that we have normalized $e$ by the ratio $H / R$.

\section{REFERENCES}

Adams, F. C., \& Bloch, A. M. 2009, ApJ, in press (arXiv:0906.4030)

Balbus, S. A., \& Hawley, J. F. 1991, ApJ, 376, 214

Brandenburg, A. 1998, in Theory of Black Hole Accretion Disks, ed. M. A. Abramowicz et al. (Cambridge: Cambridge Univ. Press), 61

Brandenburg, A., \& Dobler, W. 2002, Computer Physics Communications, 147, 471

Brandenburg, A., Nordlund, A., Stein, R. F., \& Torkelsson, U. 1995, ApJ, 446, 741

Fragile, P. C., Anninos, P., Gustafson, K., \& Murray, S. D. 2005, ApJ, 619, 327

Fromang, S., \& Papaloizou, J. 2007, A\&A, 476, 1113

Fromang, S., Papaloizou, J., Lesur, G., \& Heinemann, T. 2007, A\&A, 476, 1123

Fromang, S., Terquem, C., \& Balbus, S. A. 2002, MNRAS, 329, 18

Gammie, C. F. 1996, ApJ, 457, 355

Goldreich, P., \& Lynden-Bell, D. 1965, MNRAS, 130, 125 
Goldreich, P., \& Tremaine, S. 1980, ApJ, 241, 425

Guan, X., Gammie, C. F., Simon, J. B., \& Johnson, B. M. 2009, ApJ, 694, 1010

Hartmann, L., Calvet, N., Gullbring, E., \& D'Alessio, P. 1998, ApJ, 495, 385

Hartmann, L., D’Alessio, P., Calvet, N., \& Muzerolle, J. 2006, ApJ, 648, 484

Haugen, N. E., \& Brandenburg, A. 2004, Phys. Rev. E, 70, 026405

Haugen, N. E. L., Brandenburg, A., \& Mee, A. J. 2004, MNRAS, 353, 947

Hawley, J. F., Gammie, C. F., \& Balbus, S. A. 1995, ApJ, 440, 742

Hayashi, C. 1981, Progress of Theoretical Physics Supplement, 70, 35

Hillenbrand, L. A. 2008, Physica Scripta Volume T, 130, 014024

Ida, S., Guillot, T., \& Morbidelli, A. 2008, ApJ, 686, 1292 (IGM08)

Ilgner, M., \& Nelson, R. P. 2006, A\&A, 445, 205

Johansen, A., \& Klahr, H. 2005, ApJ, 634, 1353

Johansen, A., Klahr, H., \& Mee, A. J. 2006, MNRAS, 370, L71

Johansen, A., Oishi, J. S., Low, M.-M. M., Klahr, H., Henning, T.,\& Youdin, A. 2007, Nature, 448, 1022

Johansen, A., Youdin, A., \& Klahr, H. 2009, ApJ, 697, 1269

Johnson, E. T., Goodman, J., \& Menou, K. 2006, ApJ, 647, 1413 (JGM06)

Kley, W., \& Crida, A. 2008, A\&A, 487, L9

Laughlin, G., Steinacker, A., \& Adams, F. C. 2004, ApJ, 608, 489 (LSA04)

Lesur, G., \& Longaretti, P.-Y. 2007, MNRAS, 378, 1471

Lissauer, J. J. 1993, ARA\&A, 31, 129

Lyra, W., Johansen, A., Klahr, H., \& Piskunov, N. 2008, A\&A, 479, 883

Matsumura, S., \& Pudritz, R. E. 2003, ApJ, 598, 645

Menou, K., \& Goodman, J. 2004, ApJ, 606, 520

Nelson, R. P. 2005, A\&A, 443, 1067 (N05)

Nelson, R. P., \& Papaloizou, J. C. B. 2004, MNRAS, 350, 849 
Nitta, S., Tanuma, S., Shibata, K., \& Maezawa, K. 2001, ApJ, 550, 1119

Ogihara, M., Ida, S., \& Morbidelli, A. 2007, Icarus, 188, 522 (OIM07)

Oishi, J. S., Mac Low, M.-M., \& Menou, K. 2007, ApJ, 670, 805 (OMM07)

Paardekooper, S.-J., \& Mellema, G. 2006, A\&A, 459, L17

Paardekooper, S.-J., \& Papaloizou, J. C. B. 2009, MNRAS, 394, 2283

Papaloizou, J. C. B., \& Terquem, C. 2006, Reports on Progress in Physics, 69, 119

Pessah, M. E., Chan, C.-k., \& Psaltis, D. 2007, ApJ, 668, L51

Rein, H., \& Papaloizou, J. C. B. 2009, A\&A, 497, 595

Sano, T., Miyama, S. M., Umebayashi, T., \& Nakano, T. 2000, ApJ, 543, 486

Sano, T., Inutsuka, S.-i., Turner, N. J., \& Stone, J. M. 2004, ApJ, 605, 321

Semenov, D., Wiebe, D., \& Henning, T. 2004, A\&A, 417, 93

Shakura, N. I., \& Sunyaev, R. A. 1973, A\&A, 24, 337

Sicilia-Aguilar, A., et al. 2006, ApJ, 638, 897

Simon, J. B., Hawley, J. F., \& Beckwith, K. 2009, ApJ, 690, 974

Tanaka, H., Takeuchi, T., \& Ward, W. R. 2002, ApJ, 565, 1257

Turner, N. J., Sano, T., \& Dziourkevitch, N. 2007, ApJ, 659, 729

Ward, W. R. 1997, Icarus, 126, 261

Williamson, J. H. 1980, Journal of Computational Physics, 35, 48 